\title{
Application of Humanistic Care in Improving Patients' Satisfaction with Nursing Service in Operating Room
}

\author{
Gui E Gao ${ }^{1}$, Ding Xin Xu${ }^{1}$, Jin Ai $\mathrm{He}^{2}$ \\ ${ }^{1}$ School of Nursing, Jinan University, Guangzhou, China \\ ${ }^{2}$ The First Affiliated Hospital of Jinan University, Guangzhou, China
}

Email address:

1283262523@qq.com (Gui E Gao), dingxin@stu2019.jnu.edu.cn (Ding Xin Xu), 1259130133@qq.com (Jin Ai He)

\section{To cite this article:}

Gui E Gao, Ding Xin Xu, Jin Ai He. Application of Humanistic Care in Improving Patients' Satisfaction with Nursing Service in Operating Room. American Journal of Nursing Science. Vol. 10, No. 4, 2021, pp. 205-208. doi: 10.11648/j.ajns.20211004.13

Received: July 1, 2021; Accepted: July 10, 2021; Published: July 15, 2021

\begin{abstract}
Objective: To observe the effect of detailed humanistic care in improving patients' satisfaction with nursing service in operating room. Methods: 100 patients who underwent surgery in the operating room of the inpatient department in a grade III-A general hospital in May 2019 (control group) and 100 patients in November 2019 (observation group) were selected to conduct a survey on their satisfaction with the nursing service in operating room. The projects with low satisfaction in the survey results of May 2019 were analyzed and corresponding intervening measures such as training all staff of operating room on humanistic knowledge, formulating guidelines for detailed humanistic care, and implementing humanistic care throughout the whole operation process were adopted. Then, comparative analysis was conducted on the survey results in May and in November. Result: Patients in the observation group were significantly more satisfied than those in the control group in terms of service awareness and attitude, pre-operation explanation and privacy protection $(\mathrm{P}<0.05)$. Conclusion: Implementing detailed humanistic care, improving the service awareness and attitude of nurses in operating room, explaining to patients before nursing procedure, and paying attention to the privacy protection of the whole operation process can improve patients' satisfaction with nursing service of operating room.
\end{abstract}

Keywords: Humanistic Care, Patients' Satisfaction, Privacy Protection

\section{Introduction}

Patients' satisfaction is an important indicator for evaluating quality of medical care and service [1], as well as an objective basis for hospitals to improve quality of service by developing patient-oriented and focusing on patients' demand [2]. The National Health and Family Planning Commission emphasized in the plan of improving medical services that "paying attention to medical humanistic care" should strengthen humanistic care for patients, enhance medical personnel's humanistic quality, and respect patients' right of privacy [3]. Humanistic care is a comprehensive physical, psychological and spiritual care for patients. Many foreign studies have confirmed that humanistic care has a significant impact on the physical and mental health of patients, which has become an important topic in health-related research [4]. Studies have shown that implementing humanistic care can enhance patients' positivity for treatment [5] and also can improve patients' satisfaction [6]. In order to improve patients' satisfaction with the operating room nursing service, the implementation of detailed humanistic care in the whole process of nursing in the operating room has achieved good results, which is reported as follows.

\section{Subjects and Methods}

\subsection{Subjects}

100 inpatients who underwent surgery in May 2019 and 100 inpatients who underwent surgery in November 2019 in in a grade III-A general hospital. The inclusion criteria are those who meet all the following conditions: (1) 72 hours after surgery; (2) The patient is conscious and can cooperate to fill in the questionnaire; The exclusion criteria are those who meet one of the following conditions: (1) Discharge within 72 hours after surgery; (2) The patient has cognition impairment who cannot cooperate; The eliminate criteria are those who do not fill questionnaires completely. 


\subsection{Methods}

\subsubsection{The Control Group}

Adopted convenience sampling method to select 100 patients who underwent surgery in the operating room of the inpatient department from May 1 to May 31, 2019. Then investigated the satisfaction of patients on the third day after surgery. The patients filled in the Questionnaire of Inpatients' Satisfaction with Nursing Service in Operating Room voluntarily. The questionnaire was compiled by quality management team of the hospital, including 10 items of technological level of operating room nurses, information checking, service awareness and attitude, privacy protection, notification and explanation before operation, waiting time for operation, etc. The investigators were three uniformly trained operating room nurses. The survey results showed that the four items with low satisfaction scores $(<80$ points) for nursing service in operating room were respectively: service awareness and attitude, privacy protection, notification and explanation before operation, and waiting time for operation.

\subsubsection{The Intervention Group}

Analyzed the causes of patients' low satisfaction according to the survey results in May and took intervening measures. Implemented the intervening measures from July and selected 100 patients who underwent surgery to conduct satisfaction survey 3 months after the implementation. The survey method and personnel were the same as those in the control group. The specific intervening measures were as follows:

Conducted humanistic care training. Invited experts from the humanistic care quality group to give lectures in the department, explaining the significance of humanistic care, when to show care during the operation, and how to take specific caring measures, so as to enhance the awareness and ability of humanistic care of operating room nurses, and put the humanistic care into the whole process of operating room nursing.

Formulated standard words for self-introduction. Collected the opinions of the members of the department to compile a concise and clear words for self-introduction (Hello, I am XXX, the nurse in the operating room. Today I will assist in your operation. From now on to the end of the operation, I will always be with you. During this period, if you have any questions, please let me know. I will try my best to help you solve them.), and trained it in the whole department. It was required to greet patients and introduce themselves first and then check the information when receiving patients in the handover area.

Clarified the occasion of privacy protection for patients. Through discussion, the members of the department listed the occasions that should be protected for privacy in the whole process of surgery, and invited the experts of the hospital quality group to make supplements. The clarified occasions and methods of privacy protection are: (1) When checking patients' information, the environment should be relatively private and the voice should not be too loud. Try not to be heard by irrelevant personnel; (2) When patients wait for surgery, try to minimize the waiting time outside the operating room; (3) When operating on private parts, shielding measures should be prepared well and reduce the unnecessary personnel; (4) Help patients get dressed at the end of the operation. If there are traces of blood or disinfectant, help patients wipe up to maintain their decency and dignity.

Improved the procedure of consecutive surgery. Optimized the original consecutive operation procedure and confirmed that the time for notifying the consecutive operation is 20 minutes before the end of the previous one. Both the operating room nurse and the ward nurse should record the notification time, the operating room number and the working number of each other in order to trace them back. Installed an internal telephone in the handover area and pasted a telephone directory of each operating room beside the telephone. When patients arrive, the nurse who sends the patients calls to inform the nurses in the operating room, so as to reduce the waiting time in the handover area.

Formulated guidelines for pre-operation explanation. Formulated standardized and detailed pre-operation explanation guidelines for common operations in the operating room, such as patient transfer, venipuncture, position placing, etc. and informed patients of the purpose of operation and how to cooperate in the operation process. Made schematic cards for the positions that are difficult for the patient to understand to make patients easy to understand and cooperate.

\subsubsection{Statistical Methods}

Use SPSS 23.0 software to conduct statistical analysis, $\mathrm{t}$ test and $\chi^{2}$ test. The inspection level $\alpha=0.05$.

\section{Results}

The control group distributed 100 questionnaires and regained 98 questionnaires in which 93 questionnaires were valid. The observation group distributed 100 questionnaires and regained 100 questionnaires in which 96 questionnaires were valid. The comparative difference in gender, age, educational background, anesthesia method and other general information between the two groups showed no statistical significance $(\mathrm{P}>0.05)$, as is seen in Table 1 .

Comparison of general data between the two groups (Table 1).

Table 1. Comparison of general data between the two groups.

\begin{tabular}{|c|c|c|c|c|c|c|c|c|c|}
\hline \multirow[b]{2}{*}{ Group } & \multirow[b]{2}{*}{ Case } & \multicolumn{2}{|c|}{ Gender } & \multicolumn{2}{|l|}{ Age } & \multicolumn{2}{|l|}{ Education } & \multicolumn{2}{|c|}{ Anesthesia Method } \\
\hline & & Male & Female & $>60$ & $<\mathbf{6 0}$ & $\begin{array}{l}\text { College degree } \\
\text { or above }\end{array}$ & $\begin{array}{l}\text { High school } \\
\text { and under }\end{array}$ & $\begin{array}{l}\text { General } \\
\text { anesthesia }\end{array}$ & $\begin{array}{l}\text { Non-general } \\
\text { anesthesia }\end{array}$ \\
\hline Control Group & 93 & 48 & 45 & 55 & 38 & 59 & 34 & 42 & 51 \\
\hline Observation Group & 96 & 51 & 45 & 49 & 47 & 63 & 33 & 50 & 46 \\
\hline $\mathrm{P}$ & & 0.577 & & 0.331 & & 0.872 & & 0.420 & \\
\hline
\end{tabular}


Patients in control group gave a mark of 77 points on the satisfaction of the operating room nursing service, while the patients in observation group gave a mark of 90.5 points on the satisfaction of the operating room nursing service after the implementation of detailed nursing measures of humanistic care. The difference was statistically significant $(\mathrm{P}<0.05)$, as is seen in Table 2.

Scores of satisfactions on operating room nursing service of patients in the two groups (Table 2).

Table 2. Scores of satisfactions on operating room nursing service of patients in the two groups.

\begin{tabular}{lll}
\hline Group & Case (person) & Marks of Satisfaction \\
\hline Control Group & 93 & $77 \pm 7.15$ \\
Intervention Group & 96 & $90.5 \pm 4.97$ \\
$\mathrm{P}$ & & 0.000 \\
\hline
\end{tabular}

After the implementation of detailed humanistic care measures, the average scores of satisfactions in observation group was higher than those in the control group in terms of the service awareness and attitude of nurses, waiting time for surgery, privacy protection, explanation before operation, etc.
The difference was statistically significant $(\mathrm{P}<0.05)$, as is shown in Table 3.

Average scores of patients' satisfaction with nursing services in all aspects of the two groups (Table 3 ).

Table 3. Average scores of patients' satisfaction with nursing services in all aspects of the two groups.

\begin{tabular}{llllll}
\hline Group & Number & Service Awareness & Waiting Time & Privacy Protection & Explanation before Operation \\
\hline Control Group & 93 & $70 \pm 8.16$ & $75 \pm 9.43$ & $67 \pm 8.01$ & $72.5 \pm 7.17$ \\
Intervention Group & 96 & $90 \pm 6.43$ & $92 \pm 5.87$ & $93 \pm 4.99$ & $91.5 \pm 5.80$ \\
P & & 0.000 & 0.002 & 0.000 & 0.000 \\
\hline
\end{tabular}

\section{Conclusion}

Patients' satisfaction with the operating room nursing service can be improved by implementing humanistic care and nursing in detail, improving service awareness and attitude of the operating room nurses towards surgical patients, explaining the operation purpose and the notes for cooperation before operation, paying attention to the privacy protection of patients in the whole process, and shortening the waiting time of the patients out of the operation room.

\section{Discussion}

Humanistic care is an important factor of affecting patients' satisfaction with operating room nursing service. Through the special improvement activities of humanistic care, the patients' satisfaction with operating room nursing service has been significantly improved. This result is consistent with the research results of Liu Hui [7] and Li Dehua [8], indicating that humanistic care is an important factor of affecting patients' satisfaction. Humanistic care emphasizes people-oriented, which not only pays attention to disease nursing, but also provides comprehensive and high-quality nursing model for patients from the perspectives of psychological status and social function [9, 10]. American scholar Tucker proposed in 1998 that care is the determinant of patients' satisfaction, which is mainly determined by the attitude and caring behavior of medical staff rather than the technique itself [11]. Through humanistic care training for operating room nurses, strengthening their service awareness and formulating detailed and practical guidelines, patients' satisfaction with operating room nursing service can be improved.

The awareness of privacy protection of operating room nurses needs to be further improved. With the development of science and technology and social progress, patients' awareness of privacy protection is becoming stronger and the demand for privacy protection is constantly increasing [12]. Operating room is a special medical location for rescuing and treating patients. There will be many and various staff involved in the treatment process, so, it is particularly important to protect patients' privacy [13]. Studies have shown that paying attention to the protection of patients' privacy is not only helpful to reduce patients' psychological pressure and maintain their dignity, but also can improve patients' satisfaction and alleviate conflicts between doctors and patients [14]. In traditional idea, the privacy protection is limited to the private parts, but the protection awareness of patient's personal information, operation information, past medical history and family history, personal decency and dignity is relatively weak [15]. We should strengthen the training on privacy protection of the operating room nurses, enhance their awareness of privacy protection, and improve professional self-discipline.

Humanistic care should develop towards the direction of specialization and individuation. With the continuous development and progress of medical technology, the division of clinical specialties is becoming more detailed. Patients with different specialties, different types of diseases, different ages and different educational backgrounds have different emphasis on the care. Therefore, humanistic care should develop toward the direction of specialization. In practice, we should fully evaluate the real demand of patients with different diseases and levels in addition to basic humanistic care, and formulate individualized humanistic care plans. 


\section{References}

[1] He Fangfang, Zhu Lijun, Gao Yanqin. Analysis of Effects of Humanistic Care in Nursing on Patients' Satisfaction [J]. Fujian Journal of Medicine, 2016, 38 (3): 173-174.

[2] Wang Jingru. Investigation and analysis on outpatient satisfaction in 4 TCM hospitals in Zhejiang province [J]. Chinese Hospitals, 2019, 23 (3): 19-21.

[3] National Health and Family Planning Commission of the People's Republic of China. Interpretation of the Action Plan for Further Improving Medical Service [EB/OL]. (2015-01-05).

[4] Meezenbroek EDJ, Garssen B, Berg MVD, et al. Measuring spirituality as a universal human experience: A review of spirituality questionnaires [J]. J Relig Health, 2012, 51 (02): 336-354.

[5] Chen Yinjuan. The influence of humanistic care atmosphere on spiritual care competence among ICU nurses [J]. Today Nurses, 201, 28 (05): 14-17.

[6] Wang Fengqin. Analysis of the Effect of Human Care in Operating Room Nursing on the Improvement of Nursing quality and Patients' Satisfaction [J]. Shanxi Medical Journal, 2021, 50 (11): 1892-1895.

[7] Liu Hui. Influence of Humanistic Care in Nursing Service on Patients' Satisfaction [D]. Xinxiang Medical University, 2014.
[8] Li Dehua. Investigation and Analysis of Humanistic Care on Improving the Experience of Inpatients [J]. West China Medical Journal, 2017, 32 (2): 8: 1262-1265.

[9] Huang Xiaochun. Clinical Application of Medical Care in the Coronary Angiography [J]. China Medicine and Pharmacy, 2017, 7: 127-130.

[10] Guo Hua. Influence of Humanistic Care Nursing on Psychological Status and Nursing Satisfaction of Patients in Imageological Examination [J]. Shanxi Medical Journal, 2021, 50 (08): 1388-1390.

[11] Cheng SH, Yang MC, Chiang TL. Patient satisfaction with and recommendation of a hospital: effects of interpersonal and technical aspects of hospital care. Int J Qual Health Care, 2003, 15 (4): 345-355.

[12] Gao Yuehua. Patients' Demands for Privacy Protection and the Countermeasures [J]. Journal of Traditional Chinese Medicine Management, 2018, 26 (11) 188-190

[13] Gong Haijiao. Problems and Changes of Operating Room Nurses in Protecting Patient's Privacy Right [J]. Chinese Medical Ethics, 2019, 32 (01): 82-85.

[14] Li Jing. Application of Privacy Protection Measures in Operating Room for Female Lithotomy Patients [J]. China Health Standard Management, 2019, 10 (15): 161-163.

[15] Lin Zhi. Investigation and Analysis on Countermeasures of Patients' Demand for Privacy Protection [J]. Journal of Traditional Chinese Medicine Management, 2016, 24 (23): $10-12$. 